\title{
Relations entre caractéristiques de retrait et densité du bois chez le pin sylvestre, le sapin pectiné et l'épicéa commun
}

\author{
JF Mazet 1, G Nepveu 2* \\ 1 ONF, DR Lorraine, 5 rue Girardet, 54000 Nancy; \\ 2 INRA, station de recherches sur la qualité des bois, Champenoux, 54280 Seichamps, France
}

(Reçu le 12 mars 1990; accepté le 23 novembre 1990)

\begin{abstract}
Résumé - Des relations entre les caractéristiques de retrait du bois et son infradensité sont recherchées pour le sapin pectiné, l'épicéa commun et le pin sylvestre. Les échantillons utilisés sont des carottes de sondage de $5 \mathrm{~mm}$ de diamètre, prélevées sur un ensemble de 928 arbres. Les relations obtenues sont significatives mais restent cependant faibles. Le retrait radial est toujours mieux lié à l'infradensité que le retrait tangentiel. L'introduction d'autres variables telles que la largeur moyenne de cerne, l'âge et la circonférence de l'arbre, n'améliore pas de manière significative les relations mises en évidence.
\end{abstract}

Pinus sylvestris / Abies alba / Picea abies / retrait du bois / infradensité

Summary - Relationships between wood shrinkage properties and wood density for Scots pine, silver fir and Norway spruce. Relationships between basic wood density, tree circumference, age and wood shrinkage properties were investigated. Increment cores were used from 928 trees; the number of trees sampled for each species is given in table I. The location of the stands in the "Vosges" department is given in figure 1. The shrinkage coefficients, representing the dimensional variation of wood when its moisture content decreases by $1 \%$, were calculated. The compression wood was taken into account as it influences the shrinkage coefficient values (table II). The results of the 3 species were compared (table III): pine wood had the highest values; the values for fir and spruce were identical. The relationship between the different shrinkage coefficients was also investigated (table V). In order to study shrinkage variability, a step-by-step multiple regression was used, with basic density, ring width, age and tree circumference as the parameters. The results are given in table VI and can be summarized as follows:

1) For all species, basic density is the variable which correlates best with all the shrinkage coefficients. Therefore basic density is always the 1st variable in the regression equation;

2) The radial shrinkage coefficient is better correlated with basic density than with volumetric or tangential shrinkage;

3) The other parameters (ring width, age and tree circumference) contribute little to the explanation of shrinkage variability.

The correlations between the shrinkage coefficients and basic density are low, especially for fir wood. Figures 3, 4 and 5 show these correlations (each shrinkage coefficient as a function of basic density). All the results are discussed with reference to the literature.

Pinus sylvestris / Abies alba / Picea abies / wood shrinkage / wood density

\footnotetext{
* Correspondance et tirés à part.
} 


\section{INTRODUCTION}

Le retrait, de même que le gonflement du bois, est une propriété importante à prendre en compte dans l'évaluation de la qualité, notamment lorsque les produits sont destinés à être utilisés en ébénisterie, en menuiserie et en structure.

En effet, le retrait conditionne les déformations des pièces durant le séchage; il peut également être responsable des problèmes rencontrés sur les éléments de bois mis en œuvre, problèmes résultant des déformations dues à des pertes ou des reprises d'humidité du bois du fait de variations hygrométriques de l'atmosphère environnante.

L'objectif de cette étude était d'apprécier la possibilité d'évaluation des caractéristiques de retrait de petits échantillons de bois (carottes de sondage) résineux (pin sylvestre, épicéa commun et sapin pectiné) par d'autres caractéristiques simples et plus faciles à mesurer.

Ce travail a donc été mené avec pour but principal la recherche de l'allégement des protocoles de mesures de qualité, afin de savoir, par exemple, s'il est possible d'estimer raisonnablement le retrait du bois à partir de son infradensité, caractéristique dont la mesure est fort aisée, complétée éventuellement par quelques indications décrivant l'arbre d'origine (largeur de cerne, âge, etc.). II n'entre donc pas dans notre propos de rechercher des interprétations causales des liaisons signalées dans la littérature ou mises en évidence dans le cadre de cette étude.

\section{MATÉRIELS ET MÉTHODES}

\section{Essences}

Dans ce travail, nous nous sommes intéressés aux 3 essences résineuses les plus abondantes dans l'Est de la France, à savoir l'épicéa commun (Picea abies L (Karst), le sapin pectiné (Abies alba Mill) et le pin sylvestre (Pinus sylvestris $\mathrm{L}$ ).

\section{Échantillonnage}

L'échantillonnage recueilli ici découle en fait de celui réalisé dans le cadre d'une étude de comparaison des propriétés de base des bois résineux dits de "Pays" avec celles des bois résineux dits du "Nord", en l'occurrence de Finlande (Nepveu et al, 1988b).

Le nombre d'arbres pris en compte pour chaque essence est indiqué au tableau 1.

La répartition des points de l'échantillonnage total a été réalisée sur 3 départements (Moselle, Vosges et Ardennes) et, à l'intérieur de chaque département, au prorota de la ressource réelle effectivement existante sur pied, afin d'en obtenir une image bien représentative. Cette ventilation (2 607 arbres) est décrite par Mazet et al (1989).

Pour l'étude des retraits présentée ici, un sous-ensemble de cet échantillonnage a été utilisé, qui concerne le seul département des Vosges.

La localisation des points échantillonnés est présentée à la figure 1 .

Ces arbres ont été choisis dans une fourchette de diamètres allant de 15 à $45 \mathrm{~cm}$. Ce sont donc principalement des bois utilisables en structure.

Remarque 1 : L'absence quasi générale d'un effet "classe de diamètre" sur les divers coefficients de retraits mesurés sur cet échantillonnage (Nepveu et al, 1988b) nous a conduits à ne pas maintenir la structuration en "gros" (diamètre $30-45 \mathrm{~cm}$ ) et en "petits" arbres telle que présentée dans le tableau I.

Remarque 2 : En dépit d'un nombre assez élevé de points d'échantillonnage (voir fig 1), il ne nous est pas apparu raisonnable de rechercher des relations par sous-populations d'arbres relevant de conditions écologiques homogènes (classe d'altitude, région géographique) car le nombre d'arbres dans certaines de ces sous-populations eût été trop faible. 
Tableau I. Nombre d'arbres pris en compte dans l'échantillonnage.

\begin{tabular}{lrrr}
\hline & Sapin & Pin Épicéa \\
\hline Petits (diamètre 15 à $25 \mathrm{~cm}$ ) & 240 & 57 & 151 \\
Gros (diamètre 30 à $45 \mathrm{~cm}$ ) & 239 & 114 & 127 \\
Total & 479 & 171 & 278 \\
\hline
\end{tabular}

DEPARTEMENT DES VOSGES
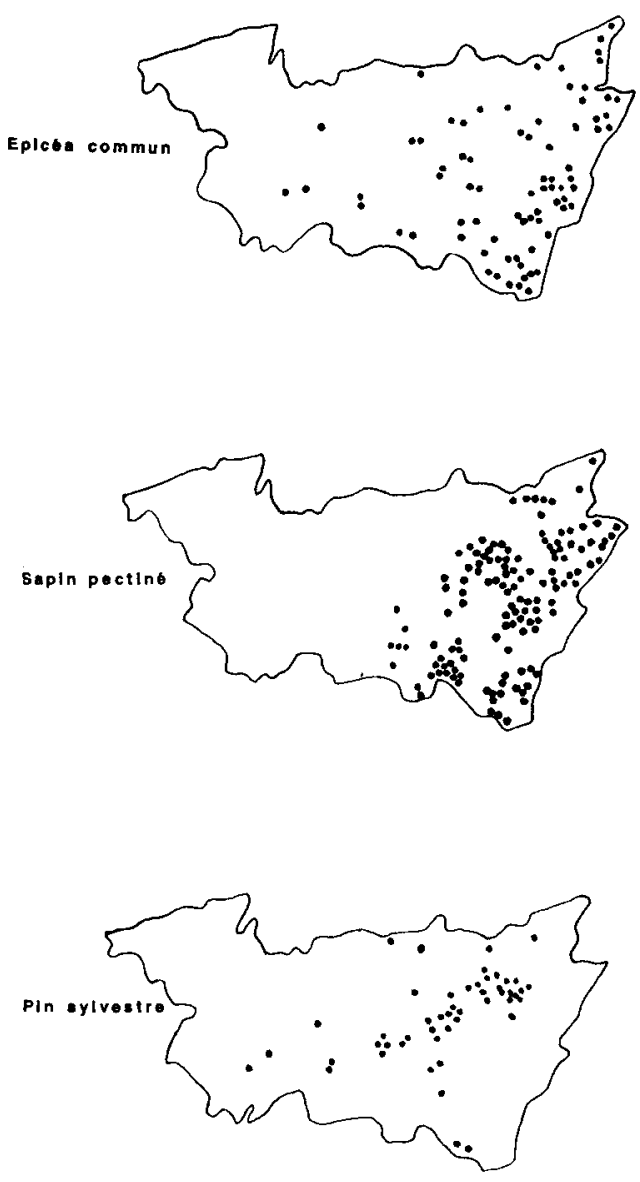

Fig 1. Localisation des placettes échantillonées dans le département des Vosges (88).

\section{Type de prélèvement effectué}

Sur chaque point (ou placette), 4 arbres ont été choisis et sondés à $1,30 \mathrm{~m}$ à la tarière de Pressler, pour en extraire 2 carottes diamétrales de $5 \mathrm{~mm}$ de diamètre perpendiculaires et passant par la moelle de l'arbre. Ces 2 carottes ont été sectionnées à la moelle donnant ainsi 4 carottes radiales par arbre.

Deux de ces carottes radiales tirées au hasard parmi les 4 carottes disponibles par arbre ont été utilisées pour la mesure des retraits et de l'infradensité du bois.

\section{Types de mesures effectuées}

Le capteur automatique unidirectionnel utilisé pour les mesures du diamètre tangentiel est décrit dans l'article de Perrin et Ferrand (1984).

Les mesures réalisées ont été :

\section{Sur carottes à l'état sec à l'air}

On a mesuré :

- le diamètre tangentiel (Diamtangsec), la valeur obtenue est la moyenne d'au moins 100 mesures (par palpage) sur l'ensemble de la carotte,

- la longueur radiale (Longsec), sur un palmer modifié (précision : $10^{-2} \mathrm{~mm}$ ),

- le poids sec à l'air des carottes (Poidssec).

\section{Après 4 j de saturation des carottes sous vide}

Ont été mesurés :

- le diamètre tangentiel (Diamtangsat),

- le longueur radiale (Longsat).

\section{Après passage des carottes à l'étuve à $102{ }^{\circ} \mathrm{C}$ pendant $24 \mathrm{~h}$}

Le poids anhydre des carottes (Poidsanh) a enfin été mesuré.

Ces données de base nous permettent de calculer : 
- l'humidité du bois lors des mesures :

$$
\mathrm{H} \%=\frac{\text { (Poidssec }- \text { Poidsanh) }}{\text { Poidsanh }}
$$

- l'infradensité du bois :

$$
I D=1 /(\text { Poidssat } \text { Poidsanh })-0,347
$$

en utilisant la formule de Keylwerth (1954).

Puis, pour l'une des 2 carottes seulement (voir aussi : "Problèmes posés par le bois de compression ):

- le coefficient de retrait tangentiel :

$$
C R T \%=\frac{(\text { Diamtangsat }- \text { Diamtangsec }) \times 100}{(\text { Diamtansat }) \times(30-\mathrm{H} \%)}
$$

- le coefficient de retrait radial :

$$
C R R \%=\frac{(\text { Longsat }- \text { Longsec }) \times 100}{(\text { Longsat }) \times(30-\mathrm{H} \%)}
$$

- le coefficient de retrait volumétrique a été estimé à partir des coefficients de retrait radial et tangentiel par la formule :

$$
C R V \%=\frac{C R T \%+C R R \%+(C R T \% \times C R R \%)}{100}
$$

Nous avons préféré utiliser les coefficients de retrait plutôt que les retraits proprement dits d'un état saturé à un état $\mathrm{sec}$ à l'air ou anhydre. Ces coefficients représentent le retrait du bois en \% lors d'une baisse de son taux d'humidité de $1 \%$, dans l'hypothèse où la déformation du bois au séchage suit une loi linéaire dans la zone hygroscopique (0-30\%).

Cette manière de procéder est conforme aux normes françaises NF B 51.004 (AFNOR, 1985a) et NF B 51.006 (AFNOR, 1985b) de déterminations de l'humidité et du retrait du bois.

Observons que nous avons admis que le point de saturation des fibres était constant et égal à $30 \%$ pour tous les échantillons. Ceci n'est pas une hypothèse trop forte (au demeurant admise par la norme française NF B $51.006)$ si l'on en croit les résultats expérimentaux obtenus sur l'épicéa commun de HauteArdenne par Fouarge et Sacre (1952).
La largeur moyenne de cerne est obtenue à partir de la circonférence à $1,30 \mathrm{~m}$ sur écorce et de l'âge de l'arbre estimé par comptage sur les carottes.

\section{Problèmes posés par le bois de compression}

Le bois de compression des résineux se caractérise par des largeurs de cernes plus fortes et par une couleur plus foncée (rougeâtre à brunâtre) que le bois normal.

La répartition du bois de compression sur la section est connue (Kollmann et Côté, 1968; Giordano, 1971) : le bois anormal est souvent localisé dans un quartier qui est en général celui présentant la plus forte excentricité.

Dans notre échantillonnage, les 2 carottes prélevées sur chaque arbre n'ont pas été orientées. Toutefois, elles ne se trouvaient jamais situées dans le même quartier de la section. II était donc peu probable que les 2 échantillons d'un même arbre présentent tous les 2 du bois de réaction.

Nous avons cherché à éliminer les arbres pour lesquels les mesures de retrait avaient été réalisées sur des carottes possédant du bois anormal.

Le bois de compression des résineux a une densité beaucoup plus forte que le bois normal provenant des mêmes arbres.

L'obtention de 2 valeurs d'infradensité par arbre nous a conduits à effectuer le rapport de la plus grande à la plus petite valeur.

Lorsque ce rapport était supérieur à 1,04 , nous pouvions soupçonner la présence de bois de compression sur la carotte possédant la plus forte valeur, et 2 cas pouvaient alors se présenter :

- soit la carotte soupçonnée de posséder du bois anormal était celle qui avait servi à obtenir les valeurs de retrait,

- soit elle ne l'était pas.

Dans le $1^{\mathrm{er}}$ cas, l'arbre était éliminé de notre étude. Dans le $2^{\theta}$ cas, l'arbre était conservé, les valeurs de retrait prises en compte et la valeur retenue pour l'infradensité était celle de la carotte ayant servi à la mesure des retraits. 
Lorsque ce rapport était inférieur ou égal à 1,04 , nous considérions qu'il n'y avait de bois de compression dans aucune des 2 carottes. Les valeurs de retrait étaient donc conservées et l'infradensité retenue était la moyenne des infradensités des 2 carottes.

La présence de bois de compression influence également les caractéristiques de retrait du bois.

II est en effet connu depuis longtemps que le retrait longitudinal du bois de compression des résineux est beaucoup plus élevé que celui du bois normal (Vintila, 1939). Kollmann et Côté (1968) signalent que si le retrait longitudinal du bois normal est pratiquement négligeable $(0,1-$ $0,2 \%$ ), celui du bois de compression peut atteindre $6-7 \%$.

Au contraire, en ce qui concerne les retraits transverses (radial et tangentiel), ces mêmes auteurs rapportent qu'ils sont en général inférieurs à ceux rencontrés pour le bois normal.

Cette indication est d'ailleurs vérifiée sur le bois de pin maritime (Polge et Illy, 1967) et sur l'épicéa de Sitka (Gentner, 1985).

Le tableau II montre les résultats obtenus en utilisant le critère de densité décrit plus haut comme indicateur de la présence de bois de compression.

Les valeurs moyennes des coefficients de rétractibilité tangentielle, radiale et volumétrique, ainsi que l'anisotropie des retraits tangentiel et radial y sont présentés pour chaque groupe et essence.

Nous observons que les valeurs des coefficients sont toujours plus faibles que les groupes "bois de compression". Cette différence de moyenne est d'ailleurs statistiquement significative en ce qui concerne les coefficients de retrait tangentiel et volumétrique pour le sapin et radial et volumétrique pour l'épicéa*.

\section{Remarque concernant le type de prélèvement effectué}

\section{Taille de l'échantillon}

Les échantillons auxquels nous nous sommes intéressés étaient donc des carottes de sondage de $5 \mathrm{~mm}$ de diamètre (grande dimension = direction radiale).

L'extrapolation des valeurs de retrait obtenues à des échantillons aux dimensions d'em-

Tableau II. Influence de la présence de bois de compression $(B C)$ sur la mesure des coefficients de retrait. Comparaison de moyennes par paires ( ${ }^{*}$ significatif à $5 \%,{ }^{* \star}$ significatif à $1 \%$, NS non significatif). Nombre de paires considérées : 63,25 et 25 pour le sapin, le pin et l'épicéa respectivement.

$\begin{array}{ccc}\text { Sapin } & \text { Pin } & \text { Épicéa } \\ \text { sans } B C \text { avec } B C & \text { sans } B C \text { avec } B C & \text { sans } B C \text { avec } B C\end{array}$

\begin{tabular}{|c|c|c|c|c|c|c|}
\hline Coefficient de retrait radial & \multicolumn{2}{|c|}{ (NS) } & \multicolumn{2}{|c|}{ (NS) } & 0,13 & 0,11 \\
\hline Coefficient de retrait tangentiel & 0,25 & 0,23 & \multicolumn{2}{|c|}{ (NS) } & \multicolumn{2}{|c|}{ (NS) } \\
\hline Coefficient de retrait volumétrique & \multicolumn{2}{|c|}{$\left({ }^{\star *}\right)$} & \multicolumn{2}{|c|}{ (NS) } & \multicolumn{2}{|c|}{$\left({ }^{*}\right)$} \\
\hline Anisotropie (ret tang/retr rad) & \multicolumn{2}{|c|}{ (NS) } & (NS) & 1,75 & \multicolumn{2}{|c|}{ (NS) } \\
\hline
\end{tabular}

\footnotetext{
* Les nombres d'arbres du tableau I constituent notre échantillonnage définitif, purgé des arbres pour lesquels les retraits avaient été mesurés sur des carottes contenant du bois de réaction. De ce fait, un point présenté figure 1 ne représente pas toujours 4 arbres.
} 
ploi ne pourra se faire qu'avec une extrême prudence.

Certains auteurs se sont intéressés à l'effet de la taille et de la forme de l'échantillon considéré sur les valeurs de retrait ou de gonflement mesurées.

Futo (1981), qui observe le retrait de coupes de bois de sapin, constate que les valeurs de retrait obtenues diminuent graduellement lorsque l'épaisseur des coupes augmente de 20 à $100 \mu \mathrm{m}$.

Signalons toutefois que Noack (1964), qui s'intéresse aux valeurs de gonflement maximal, ainsi qu'à l'anisotropie de gonflement (tang/rad) du bois d'épicéa, constate qu'il n'y a pas de différence entre des échantillons de $80 \mathrm{~mm}$ de longueur pour les directions radiale et tangentielle et $15 \mathrm{~mm}$ d'épaisseur (direction axiale) et des échantillons de $80 \mathrm{~mm}$ de longueur en direction radiale et $15 \mathrm{~mm}$ pour les directions tangentielle et axiale (forme d'échantillons donc proches de celle d'une carotte de sondage).

Enfin, les relation entre retraits sur carottes de sondage et retraits sur planches de $50 \mathrm{~cm}$ de longueur en direction axiale ont été étudiées pour l'épicéa de Sitka par Gentner (1985); les pièces provenaient de 30 arbres différents originaires de plusieurs reboisements irlandais. Une similitude apparaît dans la variation des retraits radial et tangentiel (depuis l'état vert jusqu'à l'état sec à l'air) d'un arbre à l'autre entre les 2 types d'échantillons. Toutefois, les retraits sont toujours plus forts sur les carottes.

La relation entre retrait radial sur carottes et valeur moyenne de retrait sur planches est parmi les meilleures de celles obtenues par cet auteur : $r=0,89$, significatif à $1 \%$ et 0,73 , en fonction de l'emplacement de prélèvement. En ce qui concerne le retrait tangentiel, les coefficients de corrélations linéaires sont nettement moins bons : respectivement 0,48 et 0,61 (28 ddl, significatifs à $1 \%$ ). Enfin, cet auteur note une absence de relation dans le cas du retrait axial.

\section{Représentativité de la carotte de sondage}

Rappelons ici qu'une seule carotte de sondage par arbre, prise à $1,30 \mathrm{~m}$, a été utilisée pour les mesures de retrait.

II s'avérerait donc délicat d'essayer d'étudier l'effet du sol, de la station ou de tout autre paramètre indépendant des propriétés intrinsèques de notre échantillon tel que la largeur moyenne des cernes et l'infradensité.

En effet, la variabilité intra-individuelle des caractéristiques de retrait n'est probablement pas négligeable : certains auteurs ont en effet observé des variations significatives des retraits avec la hauteur dans l'arbre, les directions de prélèvement sur une même section, ainsi que la distance radiale par rapport à la moelle chez Pinus taeda L (Yao, 1969) ou uniquement avec la hauteur chez Pinus echinata Mill (Choong et Fogg, 1989). Dans les 2 cas, les retraits radial, tangentiel et volumétrique diminuent lorsque la hauteur de prélèvement dans l'arbre augmente.

\section{RÉSULTATS ET DISCUSSION}

\section{Variabilité des valeurs de retraits comparaison des 3 essences}

Remarquons tout de suite (tableau III), que le retrait du bois est une caractéristique

Tableau III. Valeurs moyennes des coefficients de retrait obtenues pour les 3 essences (les pourcentages entre parenthèses sont les coefficients de variation).

\begin{tabular}{lcccccc}
\hline & \multicolumn{2}{c}{$\begin{array}{c}\text { Sapin } \\
479 \text { arbres }\end{array}$} & \multicolumn{2}{c}{$\begin{array}{c}\text { Pin } \\
171\end{array}$} & $\begin{array}{c}\text { Écicéa } \\
\text { arbres }\end{array}$ & 278 arbres \\
\hline Coefficent de retrait radial & 0,13 & $(21 \%)$ & 0,17 & $(24 . \%)$ & 0,13 & $(27 \%)$ \\
Coefficient de retrait tangentiel & 0,25 & $(14 \%)$ & 0,29 & $(14 \%)$ & 0,25 & $(18 \%)$ \\
Coefficient de retrait volumétrique & 0,38 & $(14 \%)$ & 0,46 & $(14 \%)$ & 0,38 & $(18 \%)$ \\
Anisotropie (retr tang/retr rad) & 2,08 & $(19 \%)$ & 1,75 & $(25 \%)$ & 2,00 & $(22 \%)$ \\
\hline
\end{tabular}



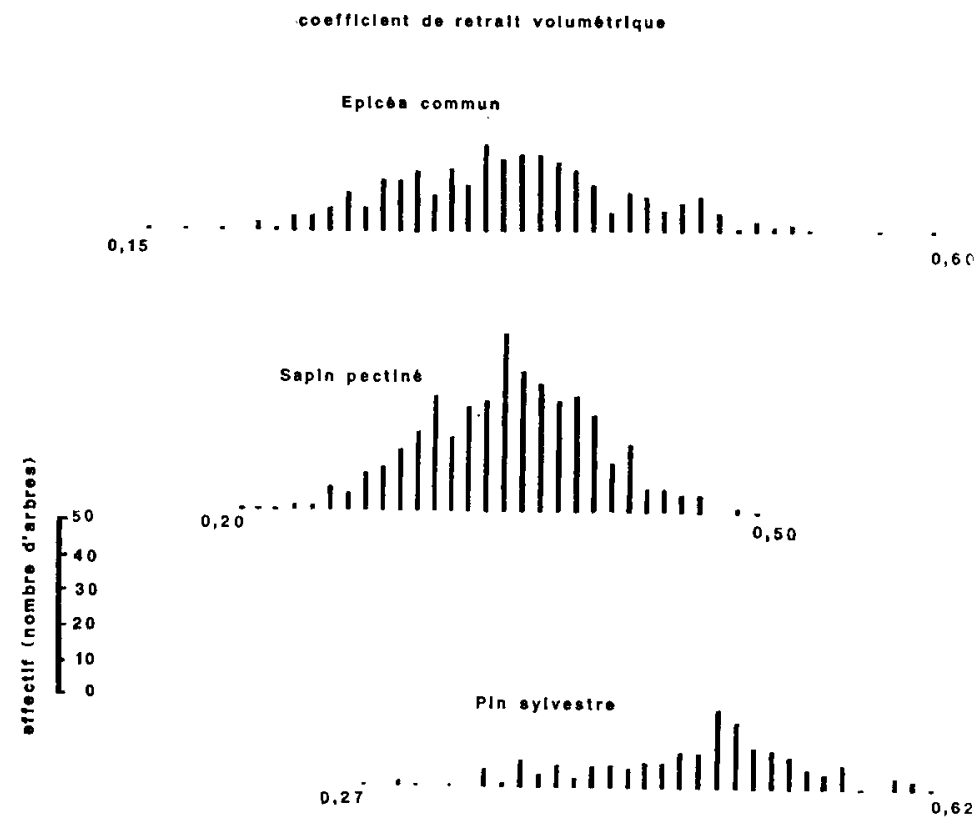

Fig 2. Variabilité du coefficient de retrait volumétrique pour les 3 essences.

très variable à l'intérieur de chacune des essences considérées (cf les coefficients de variation).

La figure 2 présente par exemple les distributions du coefficient de retrait volumétrique pour les 3 essences sous forme d'histogrammes.

Tableau IV. Valeurs moyennes des coefficients de retraits obtenues par le CTBA (CTBA, 1984).

\begin{tabular}{llll}
\hline $\begin{array}{l}\text { Coefficient } \\
\text { de retrait }\end{array}$ & Sapin & Ein \\
\hline
\end{tabular}

\begin{tabular}{lrrr} 
Radial & $0,10-0,15$ & $0,10-0,20$ & $0,10-0,15$ \\
Tangentiel & $0,25-0,35$ & $0,20-0,35$ & $0,25-0,35$ \\
Volumétrique & $0,35-0,45$ & $0,35-0,50$ & $0,40-0,45$ \\
\hline
\end{tabular}

Nous pouvons également noter que le sapin et l'épicéa présentent des valeurs moyennes extrêmement proches mais que la dispersion est beaucoup plus importante chez l'épicéa.

Le bois de pin apparaît comme étant plus sensible au retrait que celui d'épicéa ou de sapin.

À titre indicatif, le tableau IV indique les valeurs des coefficients de retrait fournies par le Centre technique du bois et de l'ameublement (CTBA, 1984). Les valeurs obtenues pour le pin sont tout à fait comparables aux nôtres. Celles concernant les coefficients tangentiel et volumétrique auraient tendance à être plus élevées que les nôtres pour le sapin et l'épicéa.

La comparaison de nos valeurs avec celles obtenues par d'autres auteurs est difficile, en raison de différences ou d'im- 
Tableau V. Corrélation entre les différents coefficients de retrait, à l'intérieur de chaque essence (" significatif à $5 \%, "{ }^{*}$ significatif à $1 \%,{ }^{* * *}$ significatif à $1 \%$, NS non significatif). CRR, CRT et CRV sont respectivement les coefficients de retrait radial, tangentiel et volumétrique; $R T / R R$ est l'anisotropie du retrait.

\begin{tabular}{|c|c|c|c|c|}
\hline & & CRR\% & CRT\% & CRV\% \\
\hline Sapin (479 arbres) & $\begin{array}{l}\text { CRT \% } \\
\text { CRV \% } \\
\text { RT/RR }\end{array}$ & $\begin{array}{r}0,42^{\star \star \star} \\
0,78^{* \star \star} \\
-0,74^{\text {}}\end{array}$ & $\begin{array}{l}0,89^{* * *} \\
0,26^{* * *}\end{array}$ & $-0,19^{* * *}$ \\
\hline Pin (171 arbres) & $\begin{array}{l}\text { CRT\% } \\
\text { CRV\% } \\
R T / R R\end{array}$ & $\begin{array}{r}0,31^{* * *} \\
0,82^{* * *} \\
-0,80^{* * *}\end{array}$ & $\begin{array}{l}0,80^{* * *} \\
0,21 * * *\end{array}$ & $-0,37^{* * *}$ \\
\hline Épicéa (278 arbres) & $\begin{array}{l}\text { CRT\% } \\
\text { CRV\% } \\
R T / R R\end{array}$ & $\begin{array}{r}0,53^{* * \star} \\
0,84^{* * *} \\
-0,75^{* * *}\end{array}$ & $\begin{array}{l}0,91^{* * *} \\
0,11^{\text {(NS) }}\end{array}$ & $-0,31 * * \star$ \\
\hline
\end{tabular}

précisions quant aux méthodologies et aux définitions des caractéristiques de retrait considérées.

\section{Relations entre les retraits}

Outre les relations entre retraits radial et tangentiel auxquelles nous pouvions nous attendre (tableau V), il est intéressant de noter que l'amplitude de l'anisotropie du retrait (rapport tang/rad) dépend beaucoup plus de la variaton de retrait radial que de celle du retrait tangentiel (un retrait radial faible peut donc laisser présager un fort comportement anisotrope du bois au cours du séchage).

\section{Recherche de facteurs explicatifs des retraits}

Comme nous l'avons déjà dit, le but principal de ce travail est la recherche d'une possibilité d'estimation du retrait du bois en fonction de paramètres simples et facilement appréhendables par le forestier à partir d'une carotte de sondage. C'est pourquoi les variables explicatives choisies sont :

- l'infradensité du bois,

- la largeur moyenne de cerne,

- l'âge,

- la circonférence de l'arbre à 1,30 m.

Pour toutes les variables à expliquer (coefficients de retraits radial, tangentiel et volumétrique, et anisotropie du retrait), et pour les 3 essences considérées, une régression progressive multiple a été effectuée en partant à chaque fois des variables explicatives citées plus haut.

Le logiciel de régression progressive multiple était celui de la programmathèque statistique STATITCF. Les variables sont introduites si leur corrélation avec la variable à expliquer est significative au moins au seuil de $5 \%$.

Les résultats de ces analyses figurent au tableau VI.

Les grandes lignes peuvent être énoncées comme suit :

- c'est l'infradensité qui est la variable la mieux corrélée aux caractéristiques de re- 


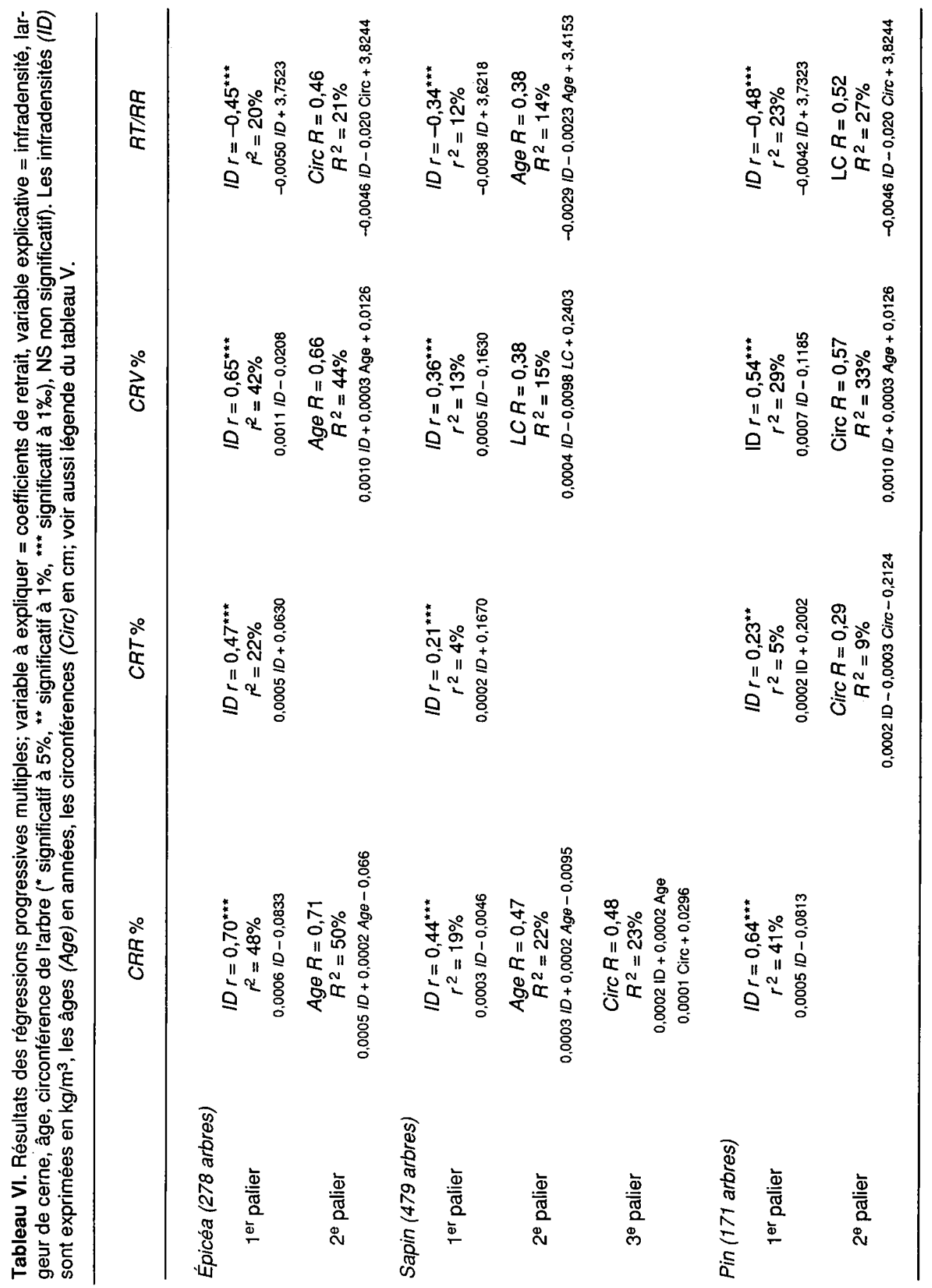


trait, et de ce fait, c'est elle qui entre au premier palier des régressions;

- c'est le coefficient de retrait radial qui est le mieux «expliqué» par l'infradensité, puis viennent dans l'ordre le coefficient de retrait volumétrique et le coefficient de retrait tangentiel.

- les autres variables (largeur moyenne de cerne, âge, circonférence) ne contribuent que très peu à améliorer le coefficient de corrélation multiple.

Ces 3 remarques doivent toutefois être modulées. En effet, s'il est vrai que l'infradensité est corrélée de manière significative avec les coefficients de retrait pour les 3 essences, la relation reste cependant faible, et ce surtout dans le cas du sapin $\left\langle R^{2}=4 \%\right.$ pour le retrait tangentiel par exemple). Nous pensons qu'il est intéressant, en complément du tableau VI, de présenter les répartitions graphiques des mesures où sont portées en ordonnées les coefficients de retrait et en abscisse l'infradensité pour l'épicéa (fig 3), le sapin (fig 4) et le pin (fig 5).

Nous constatons alors de manière claire que la déformation au séchage est beaucoup plus dépendante de l'infradensité dans la direction radiale que dans la direction tangentielle. Ce résultat est en accord avec celui obtenu par Choong (1969), ainsi qu'avec ceux de Trénard et Guéneau (1977). Ces derniers auteurs, qui cherchaient les relations liant le retrait (états saturé à anhydre) à des caractéristiques anatomiques et densitométriques pour le sapin (5 arbres, 3 échantillons par arbre, dimensions $L \times R \times T$ respectivement 100 $\times 25 \times 25 \mathrm{~mm}$ ), n'obtenaient de corrélations significatives qu'avec des variables relatives à la masse volumique et ce, uniquement pour le retrait radial. Les variables étaient en l'occurrence des valeurs moyennes sur plusieurs cernes de :
- la quantité de bois dont la masse volumique est comprise entre 700 et $900 \mathrm{~g} / \mathrm{dm}^{3}$ ( $r=0,61,15$ échantillons).

Dans le cas de l'épicéa de Sitka, Gentner (1985) constate lui aussi que la relation entre infradensité (mesurée sur carottes) et retrait tangentiel (mesuré sur planches) est, comme dans notre cas, beaucoup moins bonne que celle concernant le retrait radial. Le même type de résultat est obtenu par Yao (1969) avec un pin, et par Höwecke (1987).

La relation retrait-densité du bois a déjà été pressentie et observée de longue date : en effet, Newlin et Wilson, en 1919 (cités par Kollmann et Côté, 1968) sont les premiers à avoir proposé une relation linéaire dans le cas de plusieurs espèces en mélange : $R V=28 \times I D$, où $R V$ est le retrait volumétrique total (état saturé à anhydre) et $I D$ l'infradensité exprimée en $\mathrm{g} /$ $\mathrm{cm}^{3}$. Siau (1971) annonce une relation très proche de celle-ci. Ces auteurs ainsi que ceux qui ont repris ces relations à leur compte, tel Villière (1966), signalent toutefois leur caractère très "statistique". Ceci rend périlleuse leur application «brutale", à telle ou telle essence. Cette dernière remarque est parfaitement corroborée par nos résultats puisque nous constatons (tableau VI, lignes : 1er palier) que les ajustements entre coefficient de retrait volumétrique et infradensité du bois sont relativement différents suivant les essences.

Recherchant toujours une relation entre retrait volumétrique et densité du bois, Vintila (1939) propose diverses valeurs pour le facteur constant, selon qu'il considère le bois initial ou le bois final de différentes espèces dont le sapin et le pin. Ce coefficient constant représente en fait le taux d'humidité (en \%) au point de saturation des fibres du bois. En effet, on peut imaginer que la variation dimensionnelle d'une pièce de bois soumise au séchage est équivalente à l'espace occupé par l'eau liée avant 
EPICEAS
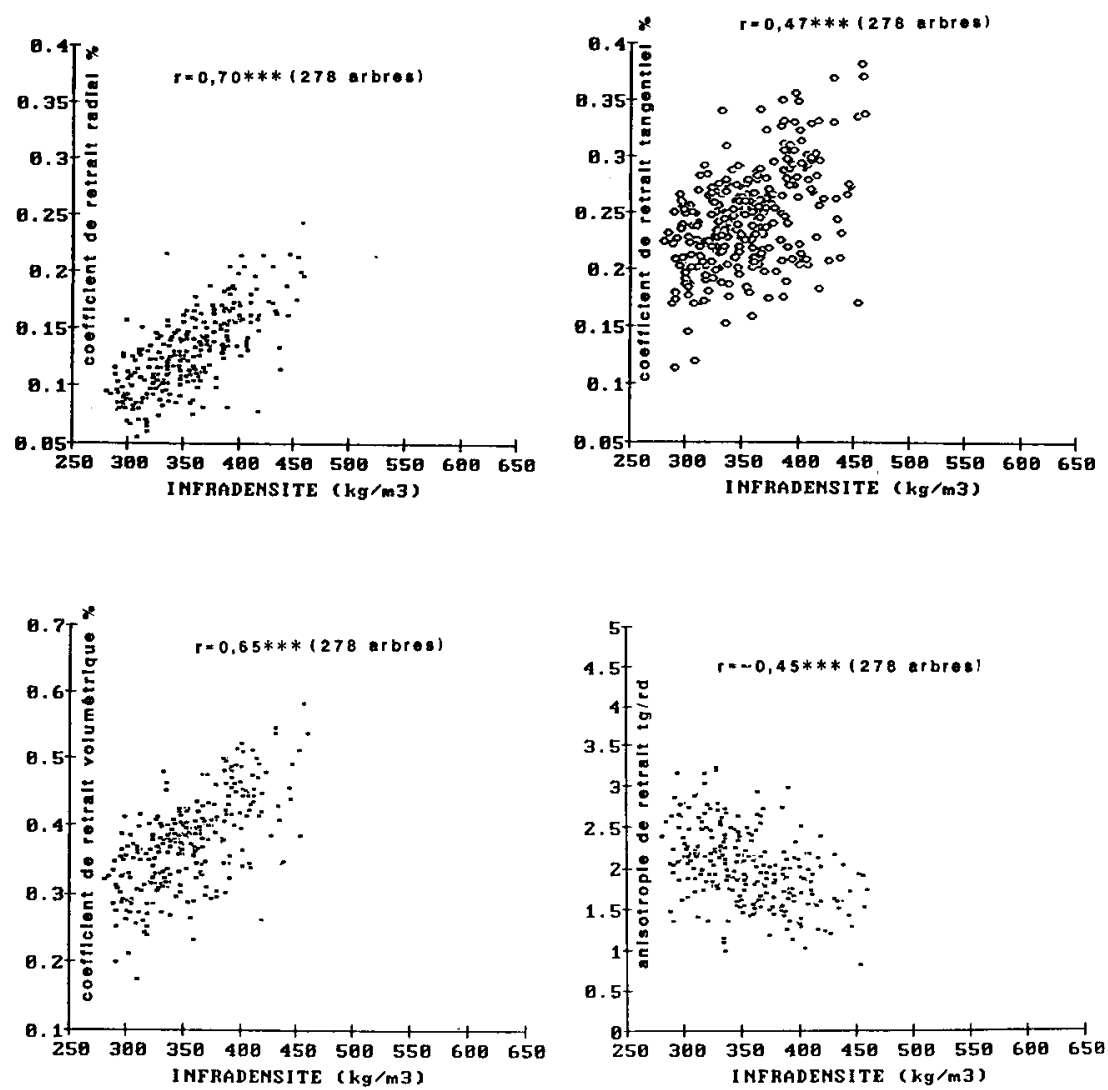

Fig 3. Répartition des valeurs des coefficients de retrait pour le bois d'épicéa commun en fonction de l'infradensité du bois.

que celle-ci n'en soit chassée (Trendelenburg, 1939; Kollmann, 1982).

Trendelenburg (1939) trouve pour l'épicéa, le sapin et le pin respectivement des coefficients constants de 32,34 et 26 . Ericson (1960) obtient, en utilisant la méthode des moindres carrés, un coefficient de 39,6 pour l'épicéa :

- la masse volumique maximale $(r=0,67$, 15 échantillons),
- et, de façon moindre, la masse volumique moyenne ( $r=0,49,15$ échantillons).

L'absence d'influence de la largeur de cerne sur les retraits, une fois enlevée celle de l'infradensité, a déjà été signalée par Yao (1969) pour Pinus taeda L.

Par contre, Kärkkäinen et Marcus (1985) comparent pour l'épicéa de Finlande du Nord des ajustements linéaires expliquant le retrait volumétrique par la 
SAPINS
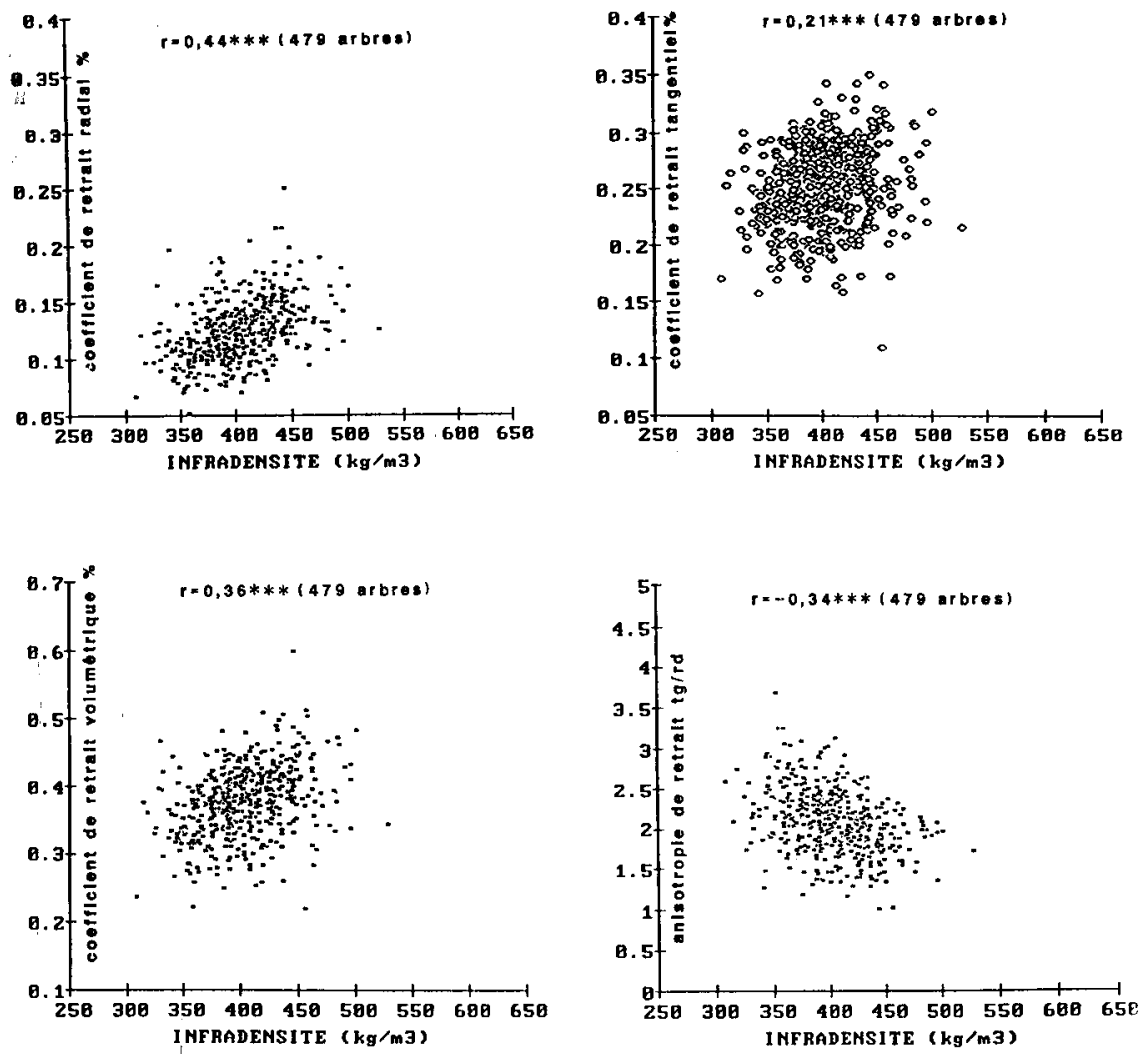

Fig 4. Répartition des valeurs des coefficients de retrait du bois de sapin pectiné en fonction de l'infradensité du bois.

densité du bois pour différentes populations définies en fonction de leur largeur moyenne de cerne $(0,5$ et 1 , puis $2 \mathrm{~mm})$. Ces auteurs observent qu'à densité constante, plus la largeur de cerne augmente, plus le retrait augmente.

\section{CONCLUSION}

Les résultats obtenus montrent bien le caractère audacieux d'une démarche qui consisterait à vouloir prédire avec précision l'amplitude des retraits du bois de ré- sineux à partir d'une mesure de l'infradensité. Toutefois, dans certains cas, notamment dans celui de l'épicéa commun, cette mesure de densité peut donner une indication utile quant à la valeur du retrait radial. En ce qui concerne le retrait tangentiel, de loin le plus important, il semble difficile de s'affranchir d'une mesure directe.

D'autre part, les différences observées entre les 3 essences quant à l'importance des liaisons ne peuvent pas être expliquées avec les données dont nous disposons. Une bonne compréhension des phé- 
PINS
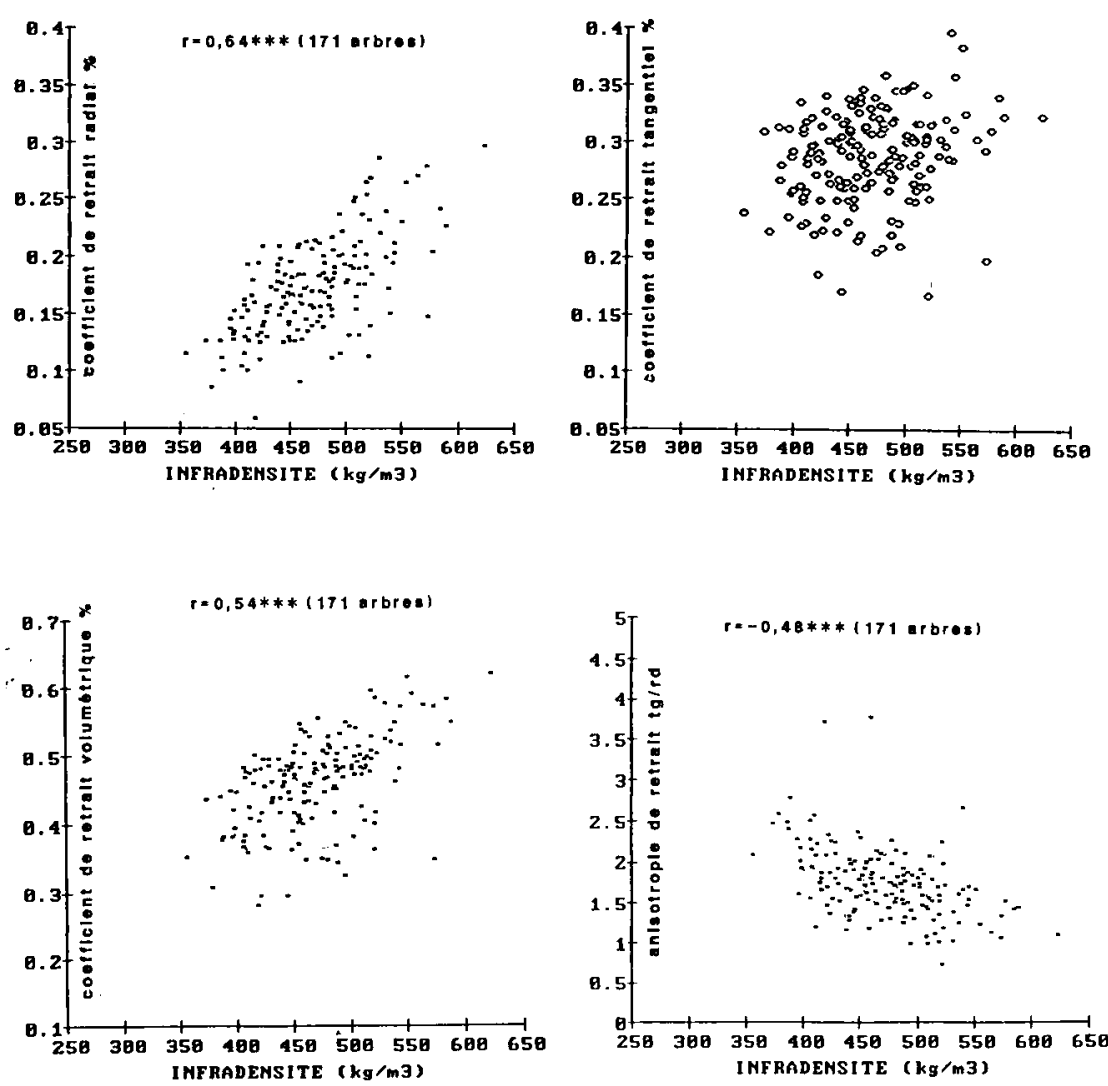

Fig 5. Répartition des valeurs des coefficients de retrait du bois de pin sylvestre en fonction de l'infradensité du bois.

nomènes de retrait des bois résineux devra sans aucun doute passer par des études anatomiques ou des analyses fines de répartition de densité à l'intérieur des cernes. Le travail de Nepveu et al (1988a), qui constitue un 1 er pas dans ce sens, révèle des différences importanies entre les profils microdensitométriques du cerne moyen des 3 essences.

De telles études pourraient également nous amener à définir des paramètres plus pertinents pour prédire de manière simple le retrait du bois.

\section{RÉFÉRENCES}

AFNOR (1985a) Bois, détermination de l'humidité. Norme française NF B 51.004, septembre, $3 p$

AFNOR (1985b) Bois, détermination du retrait. Norme française NR B 51.006, septembre, $5 p$

Choong ET (1969) Effect of extractives on shrinkage and other hygroscopic properties of ten Southern pine woods. Wood Fiber 1, 124133

Choong ET, Fogg PJ (1989) Differences in moisture content and shrinkage between in- 
nerwood and outerwood of two Shortleaf pine trees. For Prod J 39, 13-18

CTBA (1984) Les résineux français. Cahier $n^{\circ}$ 124 du Centre Tech Bois Ameublement

Ericson B (1960) Latewood Percentage, Density and Volumetric Shrinkage in Wood of Picea abies (L) Karst $F$ virgata Jacq. A comparison with Picea abies. Rapp Instn Skogsprod Skogshögsk 2, 1-15

Fouarge J, Sacre E (1952) Analyse physique, mécanique et anatomique de bois d'Epicéa, Picea abies Karst ( $P$ excelsa Link), originaire de différentes stations de la Haute Ardenne. Bull Inst Agron Stn Rech Gembloux, Tome $X X, 3-4,223-292$

Futo PL (1978) Untersuchungen von Schwindungs- und Quellungsphänomenen im Trocknungsmikroskop. 2. Mitteilung: Konstruktive Ergänzungen am Gerät. Der gegenseitige Einfluss des Früh-und Spätholzes bei der Trocknung. Holztechnol 22, 206-211

Gentner R (1985) Appréciation non destructive de la qualité du bois d'arbre sur pied : Cas de l'Epicéa de Sitka (Picea stichensis (Bong) Carr). Mémoire de $3^{e}$ année ENITEF, INRA, St Rech Qual Bois

Giordano G (1971) Tecnologia del legno. Tome 1, La Materia Prima. Utet ?

Höwecke B (1987) Quellung und Schwindung des Holzes der Weisstanne (Abies alba Mill); Untersucht an Proben aus BadenWürttembergischen Beständen. Dissertation zur Erlangung der Doktorwürde, AlbertLudwigs Universität, Freiburg

Kärkkäinen M, Marcus M (1985) Shrinkage properties of Norway spruce wood. Silva Fenn 19, 67-72

Keylwerth R (1954) Ein Beitrag zur qualitativem Zuwachsanalyse. Holz als Roh- und Werkstoff $12,77-83$

Kollmann FP (1982) Volumenschwindung von Holz und Rohdichteeinfluss, Ursachen von Ausreissern. Holz als Roh- und Werkstoff 40, 429-432

Kollmann FP, Côté WA (1968) Principles of Wood Science and Technology. Tome 1 : Solid wood. Springer Verlag

Mazet JF, Nepveu G, Velling P (1989) Étude des effets de quelques paramètres sylvicoles et environnementaux sur la densité du bois de l'Epicéa commun, du Sapin pectiné et du Pin sylvestre dans le Nord-Est de la France. Rapport interne INRA, St Rech sur la Qual Bois, $96 \mathrm{p}$

Nepveu G, Velling P, Aubert D (1988a) Comparaison de la structure des bois de Pays (Epicéa commun, Sapin pectiné et Pin sylvestre des Vosges) et des bois du Nord (Épicéa commun, Pin sylvestre) originaires de Finlande : distribution de densité dans le cerne et régularité. Rapport interne INRA, St Rech Qual Bois, $24 \mathrm{p}$

Nepveu G, Velling P, Varcin E (1988b) Comparaison de quelques propriétés de base des bois de Pays (Épicéa commun, Sapin pectiné et Pin sylvestre des Vosges) et des bois du Nord (Épicéa commun, Pin sylvestre) originaires de Finlande. Rapport interne INRA, Stn Rech Qual Bois, 74 p

Noack D (1964) Einfluss der Probenabmessungen auf die Bestimmung der Quellmasse von Holz. Holz als Roh- und Werkstoff 5, 174-182

Perrin JR, Ferrand JC (1984) Automatisation des mesures sur carottes de sondage de la densité du bois, de son retrait et des contraintes de croissance. Ann Sci For 41, 69-86

Polge H, llly G (1967) Observations sur l'anisotropie du Pin maritime des Landes. Ann Sci For 24, 205-231

Siau JF (1971) Flow in wood. Syracuse Wood Sci Ser 1, $131 \mathrm{p}$

Trénard Y, Guéneau P (1977) Relations entre la structure anatomique et l'amplitude du retrait du bois. Holzforschung 31, 194-200

Trendelenburg $R$ (1939) Uber Fasersättigungsfeuchtigkeit, Schwindmass und Raumdichtezahl wichtiger Holzarten. Holz als Roh- und Werkstoff 2, 12-17

Villière A (1966) Séchage des Bois. Dunod, Paris

Vintila $E$ (1939) Untersuchungen über Raumgewicht und Schwindmass von Früh- und Spätholz bei Nadelhölzern. Holz als Roh- und Werkstoff 2, 345-357

Yao J (1969) Shrinkage Properties of SecondGrowth Southern Yellow Pine. Wood Sci Technol 3, 25-39 